\title{
Galaxy transformation processes in clusters
}

\author{
Uta Fritze - v. Alvensleben ${ }^{1}$, Patrick Woudt ${ }^{2}$ \\ ${ }^{1}$ Universität Göttingen, Germany, ${ }^{2}$ University of Cape Town, South Africa \\ email: ufritze@astro.physik.uni-goettingen.de
}

\begin{abstract}
When falling into a galaxy cluster, the spiral - rich field galaxy population gets transformed into the characteristic E/S0/dE/dSph - rich cluster galaxy population and this already happens at surprisingly large galactocentric radii around $\sim 3 \mathrm{R}_{\text {virial }}$. A variety of transformation processes are discussed, their respective importance and timescales. Their relations to the cluster properties, however, remain to be explored. Galaxy transformation processes, transition stages, and timescales can very well be explored by a comparison of deep multi-band imaging for a large fraction of the cluster galaxy population (down to $\mathrm{M}^{*}+3$ mag out to redshifts $z \sim 0.5$ ) and of spectroscopy of the brighter members with evolutionary synthesis models. SALT's large collecting area and field of view together with its unique $U$-band sensitivity are ideal in this respect.
\end{abstract}

Keywords. galaxies: clusters: general, evolution, interactions, starburst

In preparation of forthcoming SALT observations of galaxy populations in clusters at various redshifts and their interpretation in terms of galaxy types and transition processes/stages we have calculated a large grid of GALEV Evolutionary Synthesis models for undisturbed galaxies of various types E, S0, Sa, Sb, Sc, Sd, as well as for galaxies undergoing various kinds of transformation processes affecting their Star Formation $(=\mathrm{SF})$ histories at various stages in their evolution, i.e. at various redshifts. Our models allow to follow the time evolution of galaxy spectra - including gaseous emission in terms of continuum and lines and stellar absorption features - luminosities, and colors in several filter systems from the onset of SF through starbursts of various strengths, SF truncation (on short timescales) and strangulation (on longer timescales), and merging, until the present time, and their respective redshift evolution using a standard cosmology $\mathrm{H}_{0}=65, \Omega_{0}=0.3, \Omega_{\Lambda}=0.7$ (cf. Bicker et al. 2002, Bicker et al. 2004, Bicker \& Fritze - v. Alvensleben 2006).

Models e.g. show that strong Balmer absorption lines (e.g. $\mathrm{H} \delta$ ) are only seen after strong starbursts with rapidly declining SF rates. In these cases, the $\mathrm{H} \delta$-strong or $\mathrm{E}+\mathrm{A}$ phase starts out with blue optical colors and ends when they have already turned into red. SF strangulation in a spiral galaxy, i.e. the truncation of SF on long timescales t $\sim 3 \mathrm{Gyr}$, leads to a very slow transformation into an E/S0-type spectrum without going through an $\mathrm{H} \delta$-strong or $\mathrm{E}+\mathrm{A}$ phase.

The central regions of nearby rich galaxy clusters contain $\sim 80 \% \mathrm{~S} 0$, dE, and E galaxies. Rich clusters at redshifts $0.3 \leqslant z \leqslant 0.8$, in contrast, have much higher spiral and correspondingly lower S0 fractions similar to the field galaxy population, indicating a significant transformation of the infalling spiral-rich field galaxy population into the S0rich cluster galaxy population between $z \sim 0.5$ (corresponding to galaxy ages around 9 Gyr) and $z=0$. Multi-band photometry compared to our grid of GALEV models identified possible S0 progenitors and transformation processes: e.g. SF truncation with or without preceding starbursts in spirals earlier than Sd occurring less than $\sim 3 \mathrm{Gyr}$ ago well explains the colors and luminosities of local S0s. Late-type spirals (Sd), even after strong starbursts, can only account for the lowest luminosity S0s or dSphs - unless 


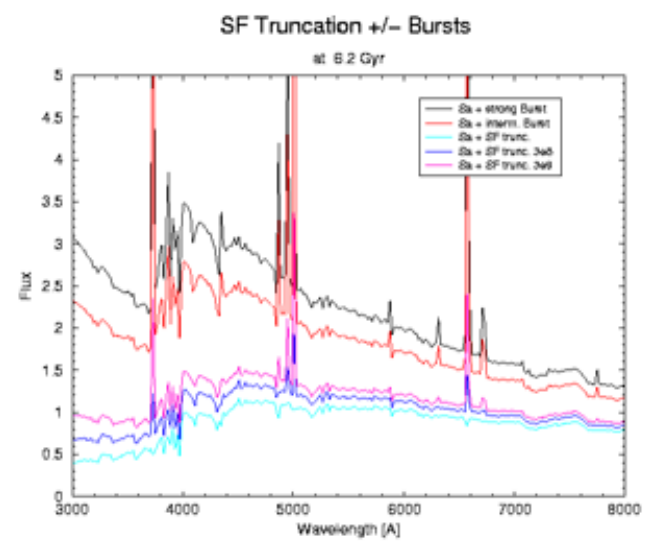

Figure 1. Sa galaxy model spectra at $z=0$ with strong and intermediate strength bursts and SF truncation on timescales $100,300,3000 \mathrm{Myr}$ occurring at an age of $6.2 \mathrm{Gyr}$, corresponding to a redshift $z \sim 1$.

involved in mergers. SF truncation in spirals earlier than $\mathrm{z} \sim 0.5-1$ would leave remnants too red for S0s by today (cf. Bicker et al. 2002).

From the luminosity evolution in different broad band filters, e.g. Johnson $U B V R I J H K$, the time or redshift evolution of Spectral Energy Distributions (SEDs) is derived. We have calculated a large grid of SEDs for galaxies of various types E. . Sd, at all redshifts, undisturbed and with a variety of effects on their SF histories like starbursts, SF truncation, and SF strangulation, setting in at various redshifts. Evolutionary and cosmological corrections as well as attenuation by intergalactic HI are consistently included, not yet included is dust extinction. A dedicated SED analysis tool has been developed in analogy to the one presented for the analysis of star clusters by Anders et al. (2004, see also Fritze - v. Alvensleben et al., this volume) for the comparison between observed multi-band galaxy SEDs and the model grid. For all galaxies with accurate multi-band photometry $(U B V R I+\mathrm{NIR}$, uncertainties $\leqslant 0.05-0.1 \mathrm{mag})$, it returns galaxy types, photometric redshifts including $\pm 1 \sigma$ uncertainties, processes and stages of transformation (Bicker \& Fritze - v. Alvensleben 2006, Bicker \& Fritze - v. Alvensleben in prep.). SALT's large collecting area allows to reach faint galaxies and dwarfs in the not too distant clusters, its large FoV allows to cover entire galaxy clusters, and its unique $U$-band sensitivity and long wavelength coverage (in particular after the NIR extension) allow for significant constraints on galaxy types, redshifts, and transformation scenarios/stages. The multi-slit option of the Robert Stobie spectrograph will allow for a detailed comparison with spectral properties for the brighter part of the cluster galaxy populations.

\section{Acknowledgements}

UFvA gratefully acknowledges travel support from the DFG under Fr 916/15-1, without which I could not have attended this conference. Part of this work was supported by the DFG under Fr 916/10-1-2.

\section{References}

Anders, P., Bissantz, N., Fritze - v. Alvensleben, U. \& de Grijs, R. 2004, MNRAS 347, 196

Bicker, J., Fritze - v. Alvensleben, U. \& Fricke, K.J. 2002, A\& A 387, 412

Bicker, J., Fritze - v. Alvensleben, U., Moeller, C.S. \& Fricke, K.J. 2004, A\&A 413, 37

Bicker, J. \& Fritze - v. Alvensleben, U. 2006, A\&A, in press 